\section{Shifting sands: adapting the multidisciplinary team model to technological and organizational innovations in cancer care}
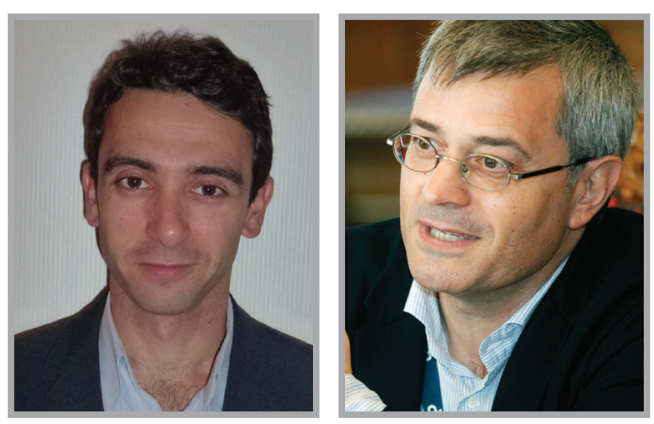

\author{
“...multidisciplinary teams \\ should be organizationally \\ reinforced and systematically \\ implemented as an essential \\ instrument for effective \\ cancer policy."
}

Joan Prades ${ }^{1,2}$ \& Josep M Borras ${ }^{*, 1,2}$

Multidisciplinary teams (MDTs) have increasingly been considered a core component of cancer care policy both at European and national levels [1,2]. While the implementation of this care model was initially motivated by its potential for a positive impact on clinical decision-making [3], the internally complex dynamics of cancer care have cemented their image as an essential tool for organizing the whole care process. Different experiences have shed light on how MDTs have evolved from isolated meetings held to make key clinical decisions, to the epicenter of a streamlined, comprehensive management approach made up of different components and interfaces. Whether they are facilitating patients' access to clinical trials to improve recruitment [4] or coordinating follow-up programs [5-7], MDTs are clearly succeeding in solving some of the most recurrent challenges of cancer care.

However, one particularly striking fact with regard to the widespread prioritization of MDTs is that it coexists with significant differences in performance, implementation and access across countries, as well as in policy measures promoting the approach [8]. Certainly, defining the functional boundaries of an expanding team may be complicated by the multiple interactions and hierarchical arrangements of professionals within organizations [9]. Pitfalls related to such situations can be found, for instance, when patients have metastatic malignant disease without an identifiable primary site, leading to clinical uncertainty and a situation of 'MDT tennis', where patients are 'bounced' between different professionals on the team [10]. More common is the involvement of professionals who are unfamiliar with patients but who nevertheless make decisions about them [11]. These experiences serve to amplify the existing shortcomings in continuity of care and suboptimal communication approaches, pointing to a need for greater structuring of MDTs.

An effective model of multidisciplinary cooperation is enabled when control over

\section{KEYWORDS}

- cancer care • multidisciplinary

- networks

'Catalonian Cancer Strategy, University of Barcelona, IDIBELL, Gran via 199, 08908-Hospitalet, Barcelona, Spain

2Department of Health, University of Barcelona, IDIBELL, Gran via 199, 08908-Hospitalet, Barcelona, Spain

*Author for correspondence: Tel.: +34 932607 820; Fax: +34 932607 316; jmborras@iconcologia.net 


\section{"Ultimately, the technological and organizational changes that are occurring in cancer care are a reflection of the dynamic progress that is being made in every corner of the field..."}

pathology-related decisions is decentralized towards MDTs. Regardless of how MDTs are formally integrated into cancer services (through the establishment of tumor boards, multidisciplinary clinics and meetings among others), their boundaries and scope of practice should be made explicit. The provision of administrative support, as well as the active promotion of team roles and 'distributed' leadership will all foster adherence to the coordination mechanisms put in place. Policy-makers and hospital managers may greatly contribute to the effectiveness and stability of this system by supporting these operating conditions, stimulating clinical accountability for the decisions made and ensuring liability on patients' clinical pathways, including in the important domain of patient-provider communication.

Recently, scientific societies, patient groups and other stakeholders involved in the European Partnership for Action Against Cancer (EPAAC) attempted to define the core elements that should be implemented by all tumor-based MDTs. A consensus group consisting of representatives from European scientific societies, patient associations and stakeholders formulated a joint policy statement on multidisciplinary cancer care [12], setting down the key elements relating to MDT goals, organization, clinical information and assessment, and patient involvement. Likewise, a new definition of MDTs was put forward: "MDTs are an alliance of all medical and healthcare professionals related to a specific tumor disease whose approach to cancer care is guided by their willingness to agree on evidence-based clinical decisions and to coordinate the delivery of care at all stages of the process, encouraging patients in turn to take an active role in their care."

The statement is intended to guide changes throughout all European health systems in a context in which the delivery of cancer care is constantly challenged by changes on the supply side, including the increased complexity of multi-modal treatments and the emergence of new areas of cancer care intervention (e.g., genetic counseling and oncogeriatrics among others). These innovations promise better patient outcomes, but they also entail changes in how healthcare professionals work. These microprocesses impact professional identities within MDTs and can have strong implications for organisational dynamics [13]. Two of the most significant contextual drivers include the emergence of cancer networks and the impact of information and communication technologies (ICT), both of which will have important effects on the MDT model of care.

To reconcile MDTs with clinical networks, the first step should be to consider them as more than just another organizational solution. On one hand, clinical complexity is growing, while clinical expertise is never evenly distributed; on the other, there are increasing policy pressures to centralize highly complex procedures and specific patient therapies [14] in order to make efficiency savings, including in approaches to clinical research from the front line of care. Since professionals, rather than managers, are the operational nodes arranging the daily life of interorganizational linkages, their ability to cooperate as a multidisciplinary team in their own hospital becomes critical for subsequent clinical collaboration with others at a network level. Cancer networks are, to a great extent, population-accountable systems made up of professionals working at different levels of clinical complexity [15]; therefore, multidisciplinary cancer care, both as a formal mechanism and as a common cultural background, can be a key lever to trigger collaborative networking. This rationale was behind the inclusion of multidisciplinary care as a core condition for the centers making up European reference networks in healthcare (Directive 2011/24EU). The importance of MDT care was also highlighted early on in the initial experience with cancer networks. Indeed, the network management team (multidisciplinary by definition) was placed in a central position [16].

The second driver of changing organizational dynamics is the spread of ICT in day-to-day clinical practice, for example in the use of online video conferencing technology to overcome logistical barriers in connecting care team members [17]. MDTs clearly stand to benefit from ICT, especially when attendance at meetings depends on connecting professionals in different hospitals or networks. However, even considering MDTs as just a formal mechanism, and not as a comprehensive area for clinical management as we propose, ICT cannot replace what constitutes the raison d'être of MDTs: the reflective, open communication on a single patient among professionals with different backgrounds, experiences and intensities of ties. The proposal to use social media to stimulate real-time decision-making [18] is fallacious if the associated parameters impair 
'thinking aloud' [19] or hinder the creativity and mutual trust that are so necessary to effective team communication. As Valdagni stated, a true team is more than "just a number of individuals digitally connected" [20]. We see as potentially dystopian a situation in which Twitter mediates MDT communication, reducing decision-making time and costs, but denying the opportunity for face-to-face interaction and the crossfertilization of different specialist cultures.

Ultimately, the technological and organizational changes that are occurring in cancer care are a reflection of the dynamic progress that is being made in every corner of the field, and MDTs have been immensely important in translating that dispersed knowledge into an integrated, coherent and personalized treatment regimen. However, the goals and functions that are increasingly assumed by MDTs, with differing degrees of professional involvement, should be actively managed. Personalizing cancer care implies defining MDTs as a specific setting for clinical decision-making management, because MDTs, regardless of how they are formally articulated, are the only way to incorporate the goals and needs of different scientific and care domains and to forge partnerships between expert professionals beyond the boundaries of their own individual services. Rebuilding the institutional logic of cancer services implies aligning different organizational approaches for example, case management, tumor boards and cancer networks - in order to undercut the potential for miscommunication, poor coordination between providers and fragmentation of services, pitfalls that are only too common in cancer care. As one of the care structures with the most potential to achieve real responsiveness to complex patient needs, MDTs should be organizationally reinforced and systematically implemented as an essential instrument for effective cancer policy.

\section{Financial \& competing interests disclosure}

We gratefully acknowledge the role of the Spanish Network of Cancer Research Groups, based in the Carlos III Institute of Health (RD12/0036/0053), in supporting our work. The authors have no other relevant affliations or financial involvement with any organization or entity with a financial interest in or financial conflict with the subject matter or materials discussed in the manuscript apart from those disclosed.

No writing assistance was utilized in the production of this manuscript.

\section{References}

1 Gouveia J, Coleman MP, Haward R et al. Improving cancer control in the European Union: conclusions from the Lisbon round-table under the Portuguese EU Presidency, 2007. Eur. J. Cancer 44, 1457-1462 (2008).

2 Atun R, Martin-Moreno JM, Ogawa T. Analysis of National Cancer Control Programmes in Europe. London: Imperial College London Business School (2009). https://spiral.imperial.ac.uk

3 Wright FC, Vito C, Langer B, Hunter A. Expert panel on multidisciplinary cancer conference standards. Multidisciplinary cancer conferences: a systematic review and development of practice standards. Eur. J. Cancer 43, 1002-1010 (2007).

4 McNair AG, Choh CT, Metcalfe C et al. Maximising recruitment into randomised controlled trials: the role of multidisciplinary cancer teams. Eur. J. Cancer 44, 2623-2626 (2008).

5 Knowles G, Sherwood L, Dunlop MG et al. Developing and piloting a nurse-led model of follow-up in the multidisciplinary management of colorectal cancer. Eur. J. Oncol. Nurs. 11, 212-223 (2007).
6 Wiederholt PA, Connor NP, Hartig GK, Harari PM. Bridging gaps in multidisciplinary head and neck cancer care: nursing coordination and case management. Int. J. Radiat. Oncol. Biol. Phys. 69, S88-S91 (2007).

7 Brennan ME, Butow P, Marven M, Spillane AJ, Boyle FM. Survivorship care after breast cancer treatment - experiences and preferences of Australian women. Breast 20, 271-277 (2011).

8 Saini KS, Taylor C, Ramirez AJ et al. Role of the multidisciplinary team in breast cancer management: results from a large international survey involving 39 countries. Ann. Oncol. 23, 853-859 (2012).

9 Carter S, Garside P, Black A Multidisciplinary team working, clinical networks, and chambers; opportunities to work differently in the NHS. Qual. Saf. Health Care 12(Suppl. 1), i25-i28 (2003).

10 Richardson A, Wagland R, Foster R et al. Uncertainty and anxiety in the cancer of unknown primary patient journey: a multiperspective qualitative study. $B M J$ Support Palliat. Care doi:10.1136/ bmjspcare-2013-000482 (2013). (Epub ahead of print)
11 Ruhstaller T, Roe H, Thürlimann B, Nicoll JJ. The multidisciplinary meeting: an indispensable aid to communication between different specialities. Eur. J. Cancer 42, 2459-2462 (2006).

12 Borras JM, Albreht T, Audisio R et al. Policy statement on multidisciplinary cancer care: European Partnership for Action Against Cancer Consensus Group. Eur. J. Cancer 50, 475-480 (2014).

13 Molleman E, Broekhuis M, Stoffels R, Jaspers F. Consequences of participating in multidisciplinary medical team meetings for surgical, nonsurgical, and supporting specialties. Med. Care Res. Rev. 67, 173-193 (2010).

14 Haward R. Organising a comprehensive framework for cancer control. In: Responding To The Challenge Of Cancer Care In Europe. Coleman MP, Alexe D-M, Albreht T, McKee M (Eds). Institute of Public Health of the Republic of Slovenia and European Observatory on Health Systems and Policies (EOHSP), Ljubljana, Slovenia, 113-134 (2008).

15 Roy DA, Litvak E, Paccaud F. PopulationAccountable Health Networks: Rethinking Health Governance and Management. The Point Publishing, Quebec, Canada (2013) 


\section{EDITORIAL Prades \& Borras}

16 Ferlie E, Fitzgerald L, McGivern G, Dopson S, Bennett C. Making Wicked Problems Governable? The Case of Managed Networks in Health Care. Oxford University Press, UK (2013).

17 Korman H, Lanni T, Shah C et al. Impact of a prostate multidisciplinary clinic program on patient treatment decisions and on adherence to NCCN guidelines: The William

Beaumont Hospital experience. Am. J. Clin. Oncol. 36, 121-125 (2012).

18 Allen TC, Liang BA. Innovation: Tumour board-introducing real time to oncology management. Nat. Rev. Clin. Oncol. 10, 552-554 (2013).
19 Weller J, Boyd M, Cumin D. Teams, tribes and patient safety: overcoming barriers to effective teamwork in healthcare. Postgrad. Med. J. 90, 149-154 (2014).

20 Crosstalk. Are tumour boards defunct? Cancer World (2014). www.cancerworld.org 